Bull. Austral. Math. Soc.

$14 \mathrm{H} 52,51 \mathrm{~N} 35,11 \mathrm{D} 09,11 \mathrm{~B} 83$

VOL. 58 (1998) [411-421]

\title{
HERON TRIANGLES AND ELLIPTIC CURVES
}

\author{
RalPh H. BuchHolz and Randall L. RathBuN
}

In this paper we present a proof that there exist infinitely many rational sided triangles with two rational medians and rational area. These triangles correspond to rational points on an elliptic curve of rank one. We also display three triangles (one previously unpublished) which do not belong to any of the known infinite families.

\section{INTRODUCTION}

A triangle with sides denoted by $(a, b, c)$ has medians, $(k, l, m)$ say, given by

$$
k=\frac{1}{2} \sqrt{2 b^{2}+2 c^{2}-a^{2}} \quad l=\frac{1}{2} \sqrt{2 c^{2}+2 a^{2}-b^{2}} \quad m=\frac{1}{2} \sqrt{2 a^{2}+2 b^{2}-c^{2}} .
$$

All rational sided triangles with two rational medians (see [1]) are completely parametrised (up to similarity) by the following equations

$$
\begin{aligned}
a & =\left(-2 \phi \theta^{2}-\phi^{2} \theta\right)+\left(2 \theta \phi-\phi^{2}\right)+\theta+1 \\
b & =\left(\phi \theta^{2}+2 \phi^{2} \theta\right)+\left(2 \theta \phi-\theta^{2}\right)-\phi+1 \\
c & =\left(\phi \theta^{2}-\phi^{2} \theta\right)+\left(\theta^{2}+2 \theta \phi+\phi^{2}\right)+\theta-\phi
\end{aligned}
$$

for rational $\phi$ and $\theta$ such that $0<\theta, \phi<1$ and $\phi+2 \theta>1$. While the sides and two of the medians, namely $k$ and $l$, are forced to be rational this is not necessarily the case for the area. Recall that Heron's formula for the area, $\Delta$ say, of the triangle $(a, b, c)$ is given by

$$
\Delta=\sqrt{s(s-a)(s-b)(s-c)}
$$

where $s=(a+b+c) / 2$ is called the semiperimeter. We shall call any rational sided triangle with rational area a Heron triangle. In [2] the authors present numerical evidence supporting the conjecture that all the rational points on five curves $C_{1}, \ldots, C_{5}$, in the region defined by $0<\theta, \phi<1$ and $\phi+2 \theta>1$ correspond (via equations (2)) to Heron triangles with two rational medians.

Received 23rd March, 1998

Copyright Clearance Centre, Inc. Serial-fee code: 0004-9729/98 \$A2.00+0.00. 
The equations of these five elliptic curves are given by

$$
\begin{aligned}
& C_{1}: 27 \theta^{3} \phi^{3}-\theta \phi(\theta-\phi)\left(8 \theta^{2}+11 \theta \phi+8 \phi^{2}\right)-3 \theta \phi\left(5 \theta^{2}-\theta \phi+5 \phi^{2}\right) \\
& \quad-(\theta-\phi)\left(\theta^{2}+4 \theta \phi+\phi^{2}\right)-\left(3 \theta^{2}-7 \theta \phi+3 \phi^{2}\right)-3(\theta-\phi)-1=0, \\
& C_{2}: 3 \theta^{2} \phi^{2}-2 \theta \phi(\theta-\phi)-\left(\theta^{2}+6 \theta \phi+\phi^{2}\right)+1=0 \\
& C_{3}: \theta \phi(\theta-\phi)^{3}-\left(\theta^{4}+11 \theta^{3} \phi+3 \theta^{2} \phi^{2}+11 \theta \phi^{3}+\phi^{4}\right) \\
& \quad-2\left(\theta^{3}-\phi^{3}\right)+10 \theta \phi+2(\theta-\phi)+1=0 \\
& C_{4}: \theta \phi(\theta-\phi)+\theta \phi+2(\theta-\phi)-1=0 \\
& C_{5}:(\theta-1)^{3} \phi^{2}+2(\theta+1)\left(\theta^{3}+2 \theta^{2}-2 \theta+1\right) \phi+(2 \theta-1)(\theta+1)^{3}=0 .
\end{aligned}
$$

If we solve equations (2) for $\theta, \phi$ in terms of $a, b, c$ we obtain

$$
\theta_{ \pm}=\frac{c-a \pm \sqrt{2 c^{2}+2 a^{2}-b^{2}}}{a+b+c} \quad \text { and } \quad \phi_{ \pm}=\frac{b-c \pm \sqrt{2 b^{2}+2 c^{2}-a^{2}}}{a+b+c}
$$

Now we bootstrap the process by substituting from equation (2) back into equations (4) via $(a, b, c)=(a(\theta, \phi), b(\theta, \phi), c(\theta, \phi))$. The pair of equations for $\left(\theta_{-}, \phi_{+}\right)$lead to the identities $\theta_{-}=\theta$ and $\phi_{+}=\phi$. However, the other pair leads to the transformation $d: \mathbb{Q} \times \mathbb{Q} \mapsto \mathbb{Q} \times \mathbb{Q}$ given by

$$
d(\theta, \phi)=\left(\frac{\theta \phi+2 \phi^{2}-\theta-\phi-1}{3 \theta \phi+\theta-\phi+1}, \frac{-2 \theta^{2}-\theta \phi-\theta-\phi+1}{3 \theta \phi+\theta-\phi+1}\right) .
$$

(Note that this corrects the transformation given in [2].) This maps a point in the $\theta \phi$ plane to a related point which corresponds to the same triangle. (Geometrically, this amounts to simply flipping the triangle about the third median, that is, interchanging sides $a$ and $b$ and simultaneously medians $k$ and $l$.) We call such points "dual" points and under this mapping the curves $C_{1}$ and $C_{2}$ are dual as are $C_{3}$ and $C_{4}$, while $C_{5}$ is self-dual. This implies that it is sufficient to prove that all rational points on just the curves $C_{2}, C_{4}$ and $C_{5}$ say, correspond to Heron triangles with two rational medians.

Furthermore, we now show that $C_{2}, C_{4}$ and $C_{5}$ are all birationally equivalent to the same elliptic curve so we shall just prove the main theorem for curve $C_{4}$. Notice that the former three curves are quadratic in $\phi$ so that when we calculate the discriminant of each (with respect to $\phi$ ) we obtain

$$
\begin{aligned}
& \mathcal{D} i s c\left(C_{2}\right)=4\left(4 \theta^{4}+8 \theta^{3}+5 \theta^{2}-2 \theta+1\right) \\
& \mathcal{D} i s c\left(C_{4}\right)=\theta^{4}+2 \theta^{3}+5 \theta^{2}-8 \theta+4 \\
& \mathcal{D} i s c\left(C_{5}\right)=4 \theta^{2}(\theta+1)^{2}\left(\theta^{4}+2 \theta^{3}+5 \theta^{2}-8 \theta+4\right)
\end{aligned}
$$


Since we are searching for rational points on each of the curves we require the corresponding discriminants of each to be a rational square. All the rational points which force this correspond to rational points on the elliptic curve

$$
Y^{2}=X^{4}+2 X^{3}+5 X^{2}-8 X+4
$$

since we can map $X$ to $-1 / \theta$ for $C_{2}$, while for $C_{4}$ and $C_{5}$ we just map $X$ to $\theta$.

\section{Proof of RATional AREa}

In this section we prove our main result.

THEOREM 1. Every rational point on the curve

$$
C_{4}: \theta^{2} \phi-\theta \phi^{2}+\theta \phi+2 \theta-2 \phi-1=0
$$

such that $0<\theta<1$ and $0<\phi<1$ and $2 \theta+\phi>1$ corresponds to a triangle with rational sides, rational area, and two rational medians.

Outline of the proof:

(i) The inequalities for $\theta$ and $\phi$ are simply obtained from the triangle inequalities.

(ii) Reduce the squarefree part of the square of the area from degree 11 to degree 8.

(iii) Show that all but finitely many points on $C_{4}$ can be obtained from the rational points on an elliptic curve, $E$ say.

(iv) Finally we use induction in the group $E(\mathbb{Q})$. We show that any point which corresponds to a triangle with rational area leads, in all possible ways, to another point with rational area. Another way of viewing this step is that the group operation on $E$ preserves the rationality of the square root of the degree 8 polynomial (mentioned in (ii)) and hence the rationality of the area.

(i) Recall that the sides of a triangle corresponding to a point $(\theta, \phi) \in C_{4}(\mathbb{Q})$ are given by equations (2). These equations immediately imply that the sides and two medians are rational so we need only check the area $\Delta$ and whether or not $(a, b, c)$ form a proper triangle. Substituting (2) into the triangle inequalities provides the following inequalities in terms of $\theta$ and $\phi$ :

$$
\begin{aligned}
& a+b \leqslant c \Longleftrightarrow(\theta-1)(\phi+1)(\theta-\phi+1) \geqslant 0, \\
& b+c \leqslant a \Longleftrightarrow \phi(\theta+1)(2 \theta+\phi-1) \leqslant 0, \\
& c+a \leqslant b \Longleftrightarrow \theta(\phi-1)(\theta+2 \phi+1) \geqslant 0 .
\end{aligned}
$$




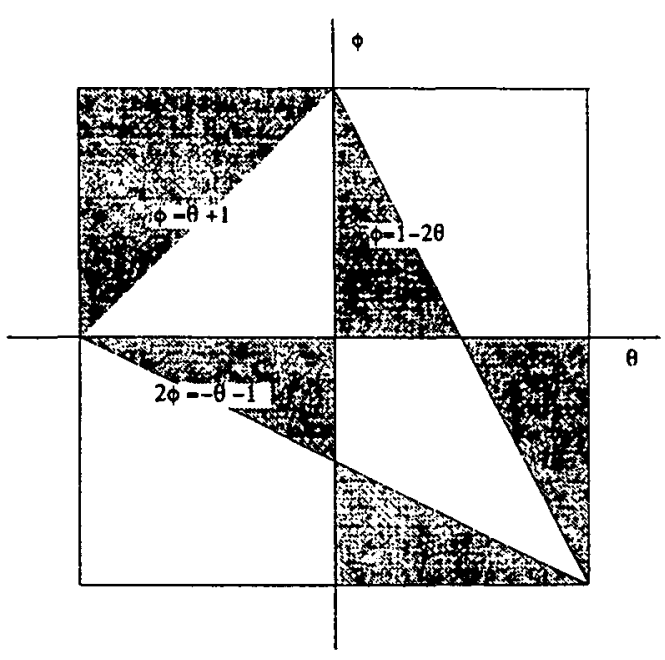

Figure 1: The excluded regions of the $\theta \phi$-plane

These define excluded regions in which proper triangles cannot form. The remaining four regions contain copies of the same set of triangles and so it is sufficient to just consider one of them, for example the one in the positive quadrant.

(ii) Again using equations (2), in Heron's formula this time, we can calculate the area of the corresponding triangle as $\Delta=\sqrt{g(\theta, \phi)}$ where

$$
g(\theta, \phi)=\theta \phi\left(1-\theta^{2}\right)\left(1-\phi^{2}\right)(3 \theta \phi+\theta-\phi+1)(2 \theta+\phi-1)(\theta+2 \phi+1)(\theta-\phi+1)
$$

The squarefree part of $g(\theta, \phi)$ can be reduced to degree 8 on $C_{4}$ by the following simple lemma.

LEMMA 1. Let $(\theta, \phi)$ be a point on $C_{4}(\mathbb{Q})$ and

$$
f(\theta, \phi)=\theta \phi\left(1-\theta^{2}\right)\left(1-\phi^{2}\right)(2 \theta+\phi-1)(\theta+2 \phi+1)
$$

Then $g(\theta, \phi)$ is a rational square if and only if $f(\theta, \phi)$ is a rational square.

Proof: The curve $C_{4}$ is equivalent to $\theta-\phi+1=3 /(\theta \phi+2)$ which when substi- 
tuted into $g(\theta, \phi)$ gives

$$
\begin{aligned}
g(\theta, \phi) & =\theta \phi\left(1-\theta^{2}\right)\left(1-\phi^{2}\right)\left(3 \theta \phi+\frac{3}{\theta \phi+2}\right)(2 \theta+\phi-1)(\theta+2 \phi+1) \frac{3}{(\theta \phi+2)} \\
& =\theta \phi\left(1-\theta^{2}\right)\left(1-\phi^{2}\right)(\theta \phi(\theta \phi+2)+1)(2 \theta+\phi-1)(\theta+2 \phi+1) \frac{3^{2}}{(\theta \phi+2)^{2}} \\
& =\theta \phi\left(1-\theta^{2}\right)\left(1-\phi^{2}\right)(2 \theta+\phi-1)(\theta+2 \phi+1)\left[\frac{3(\theta \phi+1)}{(\theta \phi+2)}\right]^{2} \\
& =f(\theta, \phi)\left[\frac{3(\theta \phi+1)}{(\theta \phi+2)}\right]^{2} .
\end{aligned}
$$

(iii) At this stage it is sufficient to prove that all rational points in $C_{4}(\mathbb{Q})$ preserve $f(\theta, \phi)=r^{2}$ for some $r \in \mathbb{Q}$. To describe all rational points on $C_{4}$ we note that the mapping $a: E \mapsto C_{4}$ defined by

$$
a(X, Y)=\left(\frac{2 X^{3}+X^{2}-8 X+4-Y^{2}-2 Y}{(3 X-2+Y)(X+Y)}, \frac{2 X^{3}-5 X^{2}+2 X-Y^{2}-2 X Y}{(3 X-2+Y)(X+Y)}\right)
$$

leads to the elliptic curve

$$
E: Y^{2}+X Y=X^{3}+X^{2}-2 X
$$

which has rank 1 and torsion subgroup isomorphic to $\mathbb{Z} / 2 \mathbb{Z}$. The group of rational points is given by $E(\mathbb{Q})=\langle(0,0),(2,2)\rangle$ (see $[3]$ ) where $(0,0)$ is the order 2 generator and $(2,2)$ is the torsion-free generator.

Now we find that all except a finite number of rational points on $E$ correspond to rational points on $C_{4}$ and vice versa. Clearly, the rational points on $E$ given by

$$
(X, Y)=(-2,2),(0,0),(1,-1) \text { and }(2,-4)
$$

are all the singularities of the mapping $a(X, Y)$ and so all other rational points, on $E$, correspond to rational points on $C_{4}(\mathbb{Q})$.

Furthermore, the only rational points which may be missed on $C_{4}$ are

$$
(\theta, \phi)=(1,1),(-1,-1)
$$

which are obtained from the singularities of the inverse mapping $a^{-1}: C_{4} \mapsto E$ given by

$$
a^{-1}(\theta, \phi)=\left(\frac{2\left(\theta \phi-\phi^{2}+\phi+1\right)}{(\theta-\phi)^{2}}, \frac{2\left(2-\theta^{2}-\theta-2 \phi^{2}+3 \phi+3 \theta \phi-\phi(\theta-\phi)^{2}\right)}{(\theta-\phi)^{3}}\right)
$$


However, it turns out that $(\theta, \phi)=(-1,-1)=a(1,0)$, while using homogeneous coordinates one finds that $(\theta, \phi)=(1,1)$ corresponds to $(X, Y)=(1,-1)$. We shall ignore the latter fact and just treat $(\theta, \phi)=(1,1)$ separately when the time comes.

(iv) The final part of the proof requires the following three lemmas. The first one covers the case of negation of points on $E$.

LEMMA 2. If $(X, Y) \in E(\mathbb{Q})$ such that $f(a(X, Y))=r^{2}$ and $\left(X^{\prime}, Y^{\prime}\right)=-(X, Y)$ then $f\left(a\left(X^{\prime}, Y^{\prime}\right)\right)=R^{2}$.

ProOF: First we use $E$ to remove all powers of $Y$ greater than the first from the squarefree part of $f(a(X, Y))$ to get

$$
f(a(X, Y))=\left(\frac{8 X(X-1)(X-2)(X+1)}{(3 X+Y-2)^{4}(X+Y)^{4}}\right)^{2}\left(p_{7}(X) \cdot p_{6}(X) \cdot Y+p_{15}(X)\right)
$$

where

$$
\begin{aligned}
p_{7}(X)= & 12 X^{7}-96 X^{6}-96 X^{5}+444 X^{4}-161 X^{3}-276 X^{2}+204 X-32 \\
p_{6}(X)= & 2 X^{6}-8 X^{5}-70 X^{4}+102 X^{3}+45 X^{2}-108 X+36 \text { and } \\
p_{15}(X)= & 4 X^{15}+21 X^{14}-984 X^{13}+2918 X^{12}+17862 X^{11}-37355 X^{10} \\
& \quad-58326 X^{9}+166369 X^{8}-25775 X^{7}-194595 X^{6}+153070 X^{5} \\
& +26088 X^{4}-80512 X^{3}+37392 X^{2}-6432 X+256 .
\end{aligned}
$$

Next, note that on $E$ we have $\left(X^{\prime}, Y^{\prime}\right)=(X,-X-Y)$ so that we get

$$
f\left(a\left(X^{\prime}, Y^{\prime}\right)\right)=\left(\frac{8 X(X-1)(X-2)(X+1)}{(2 X-Y-2)^{4} Y^{4}}\right)^{2}\left(-p_{7}(X) \cdot p_{6}(X) \cdot Y+q_{15}(X)\right)
$$

where $q_{15}(X)=p_{15}(X)-X \cdot p_{7}(X) \cdot p_{6}(X)$. Now, rather surprisingly, when we multiply the two squarefree parts of $f(a(X, Y))$ and $f\left(a\left(X^{\prime}, Y^{\prime}\right)\right)$ together we obtain a perfect square, namely

$$
\left(p_{7}(X) \cdot p_{6}(X) \cdot Y+p_{15}(X)\right)\left(-p_{7}(X) \cdot p_{6}(X) \cdot Y+q_{15}(X)\right)=\left[r_{15}(X)\right]^{2}
$$

where $r_{15}(X)=(4 X+1)(X-8)(X+1)(X+2)^{2}(X-2)^{3}(X-1)^{7}$. Clearly, we now have $f\left(a\left(X^{\prime}, Y^{\prime}\right)\right)=K^{2} / f(a(X, Y))$ and so the assumption that $f(a(X, Y))=r^{2}$ proves lemma 2.

Now we need to consider what happens when we add the infinite order generator (namely $(2,2)$ ) to a point on $E(\mathbb{Q})$. 
LEMma 3. If $(X, Y) \in E(\mathbb{Q})$ and $\left(X^{\prime}, Y^{\prime}\right)=-\{(X, Y)+(2,2)\}$ then there exists $R \in \mathbb{Q}$ such that $f\left(a\left(X^{\prime}, Y^{\prime}\right)\right)=R^{2}$.

Proof: First we intersect the line through $(X, Y)$ and $(2,2)$ with $E$ to find that

$$
\left(X^{\prime}, Y^{\prime}\right)=\left(\frac{2 X^{2}+4 X-4-6 Y}{(X-2)^{2}}, \frac{-4 X^{3}-18 X^{2}+12 X+8+18 X Y}{(X-2)^{3}}\right)
$$

for all $(X, Y)$ except $(2,2)$. Substituting this into the expression for $f\left(a\left(X^{\prime}, Y^{\prime}\right)\right)$ and using $E$ to reduce powers of $Y$ leads to

$$
f\left(a\left(X^{\prime}, Y^{\prime}\right)\right)=\left(\frac{3 X(X-1)(X-2)(X+2)(X+1)^{2}}{(X+1)^{4}}\right)^{2} .
$$

Note that $-2 *(2,2)=(1,0)$ on $E$ and so we have $f(a(-\{(2,2)+(2,2)\}))=$ $f(a(1,0))=f(-1,-1)=0^{2}$.

Combining Lemma 2 with Lemma 3 immediately implies that $(X, Y)+(2,2)$ also preserves the rationality of the area.

Finally, we need to ensure that adding the order two point to any point on $E(\mathbb{Q})$ preserves the rationality of the area. To ease the proof we use that fact that on $E$ we have $(0,0)+(2,2)=(-1,2)$ which leads to the identity

$$
(x, y)+(0,0)=-\{-\{(x, y)+(-1,2)\}+(2,2)\} .
$$

Thus it is sufficient to check addition of the point $(-1,2)$ to arbitrary points on $E(\mathbb{Q})$.

Lemma 4. If $(X, Y) \in E(\mathbb{Q})$ and $\left(X^{\prime}, Y^{\prime}\right)=-\{(X, Y)+(-1,2)\}$ then there exists $R \in \mathbb{Q}$ such that $f\left(a\left(X^{\prime}, Y^{\prime}\right)\right)=R^{2}$.

Proof: First we intersect the line through $(X, Y)$ and $(-1,2)$ with $E$ to find that

$$
\left(X^{\prime}, Y^{\prime}\right)=\left(\frac{-X^{2}-5 X+2-3 Y}{(X+1)^{2}}, \frac{-X^{3}+3 X^{2}+18 X-4+9 Y}{(X+1)^{3}}\right)
$$

for all $(X, Y)$ except $(-1,2)$. Now, as before we substitute this into the expression for $f\left(a\left(X^{\prime}, Y^{\prime}\right)\right)$ and use $E$ to reduce powers of $Y$ to obtain

$$
f\left(a\left(X^{\prime}, Y^{\prime}\right)\right)=\left(\frac{24(X-1)(X+2)(X+1)}{(X-2)^{2} X^{2}}\right)^{2} .
$$

Note that $-2 *(-1,2)=(1,0)$ on $E$ and so we have $f(a(-\{(-1,2)+(-1,2)\}))=$ $f(a(1,0))=f(-1,-1)=0^{2}$. 
These three lemmata imply that negation of points and addition of either generator of $E(\mathbb{Q})$ preserves the property that $f(a(X, Y)) \in \mathbb{Q}^{2}$. Since $f(a(-1,2))=f(-1,3)=$ $0^{2}$ and $f(a(2,2))=f(0,-3 / 2)=0^{2}$ we conclude that all points on $E(\mathbb{Q})$ preserve $f(a(X, Y)) \in \mathbb{Q}^{2}$. Hence all points $(\theta, \phi) \in C_{4}(\mathbb{Q})$ preserve $f(a(X, Y)) \in \mathbb{Q}^{2}$ except perhaps $(\theta, \phi)=(1,1)$, as noted earlier. However $f(1,1)=0^{2}$. Thus the main theorem is proven.

\section{INFINITELY MANY HERON-2-MEDIAN TRIANGLES}

The approach we use to show that there are infinitely many Heron triangles with two rational medians is simply to observe that an unbounded portion of the positive arm of the elliptic curve $E$ is mapped into the bounded region defined by $0<\theta, \phi<1$ and $2 \theta+\phi>1$. Then we use the fact (established in Lemma 5) that the rational points on the curve, namely $E(\mathbb{Q})$, are dense in the group of real points, $E(\mathbb{R})$.

LEMMA 5. If $(X, Y)$ is an infinite order point on the unbounded component of an elliptic curve $E$ then $E(\mathbb{Q})$ contains points such that $|X|$ is arbitrarily large.

Proof: Suppose that the infinitely many rational points are contained in a bounded region, that is, $X_{\min }<X<X_{\max }, Y_{\min }<Y<Y_{\max }$ for all $(X, Y) \in E(\mathbb{Q})$.

First we argue that $E(\mathbb{R})$ contains no limit points (that is, a possibly real point on the curve which is the limit of a sequence of rational points on the curve). If it did contain a sequence $\left\{P_{n}\right\}$ converging to $P$ say, then the sequence $\left\{-P_{n}\right\}$ converges to $-P$ and the chords formed by the points $P_{n}$ and $-P_{n-1}$ have increasingly large slope. These slopes are not necessarily monotonically increasing but for sufficiently large $n$ we shall find, by adding the points $P_{n}$ and $-P_{n-1}$ on the curve, that

$$
\left|X\left(P_{n}-P_{n-1}\right)\right|>X_{\max }
$$

which contradicts our initial assumption.

With no limit points there must be a minimum distance, $\delta$ say, between any two rational points on $E$. But with a finite arc length of the curve $E$ in the bounded region we end up with only a finite number of rational points on $E$ contradicting the fact that $E(\mathbb{Q})$ has rank at least one.

Using this result and the observation that $E(\mathbb{Q})$ does contain points on the bounded component (for example $(0,0)$ ) is essentially enough to show that $E(\mathbb{Q}$ ) is dense in $E(\mathbb{R})$. (It is an interesting question to determine the density of the rational points in the group of real points for arbitrary curves.) However, the Lemma above is sufficient for our current needs.

THEOREM 2. The curve

$$
C_{4}: \theta^{2} \phi-\theta \phi^{2}+\theta \phi+2 \theta-2 \phi-1=0
$$


contains an infinite number of rational points in the region $0<\theta<1,0<\phi<1$ and $2 \theta+\phi>1$.

Proof: Recall the mapping from $E$ to $C_{4}$ defined by

$$
a(X, Y)=\left(\frac{2 X^{3}+X^{2}-8 X+4-Y^{2}-2 Y}{(3 X-2+Y)(X+Y)}, \frac{2 X^{3}-5 X^{2}+2 X-Y^{2}-2 X Y}{(3 X-2+Y)(X+Y)}\right) .
$$

Setting $a(X, Y)=(\theta, \phi)$, we find that the inequalities $\theta>0, \phi>0, \theta<1, \phi<1$ and $2 \theta+\phi>1$ respectively lead to

$$
\begin{aligned}
Y^{2}+2 Y & <2 X^{3}+X^{2}-8 X+4 \\
Y^{2}+2 X Y & <2 X^{3}-5 X^{2}+2 X \\
Y^{2}+2 X Y & >X^{3}-X^{2}-3 X+2 \\
Y^{2}+(3 X-1) Y & >X^{3}-4 X^{2}+2 X \\
2 Y^{2}+(3 X+1) Y & <3 X^{3}-3 X^{2}-6 X+4
\end{aligned}
$$

Now by solving each of these as a quadratic in $Y$ one obtains

$$
\begin{aligned}
& Y<Y_{1}^{ \pm}=-1 \pm \sqrt{2 X^{3}+X^{2}-8 X+5} \\
& Y<Y_{2}^{ \pm}=-X \pm \sqrt{2 X^{3}-4 X^{2}+2 X} \\
& Y>Y_{3}^{ \pm}=-X \pm \sqrt{X^{3}-3 X+2} \\
& Y>Y_{4}^{ \pm}=1 / 2-3 X / 2 \pm \sqrt{X^{3}-7 X^{2} / 4+X / 2+1 / 4} \\
& Y<Y_{5}^{ \pm}=-1 / 4-3 X / 4 \pm \sqrt{3 X^{3} / 2-15 X^{2} / 16-21 X / 8+33 / 16} .
\end{aligned}
$$

We consider the positive arms of each curve. Notice that the radical part dominates each of the $Y_{i}$ for large $X$ and that $Y_{2}^{+}(8)=Y_{5}^{+}(8)=20$. A number of comparisons (similar to the ones shown below) lead to the ordering

$$
Y_{4}^{+}<Y_{3}^{+}<Y_{5}^{+}<Y_{2}^{+}<Y_{1}^{+} \text {for all } X>8
$$

on these curves. In fact, the inequalities above force $Y_{3}^{+}<Y<Y_{5}^{+}$for all $X>8$. So the aim now is to show that an infinite part of the elliptic curve $E$ is contained in the region of the $X Y$-plane defined by $Y_{3}^{+}$and $Y_{5}^{+}$. Solving $E$ for $Y$ in terms of $X$ leads to

$$
Y_{E}^{ \pm}=-X / 2 \pm \sqrt{X^{3}+5 X^{2} / 4-2 X} \text {. }
$$

Hence we calculate

$$
\begin{aligned}
Y_{E}^{+}-Y_{3}^{+} & =X / 2+\sqrt{X^{3}+5 X^{2} / 4-2 X}-\sqrt{X^{3}-3 X+2} \\
& >\sqrt{X^{3}+X^{2}-2 X}-\sqrt{X^{3}-3 X+2} \\
& =\sqrt{X(X-1)(X+2)}-\sqrt{(X-1)^{2}(X+2)} \\
& >\sqrt{X}-\sqrt{X-1} \\
& >0
\end{aligned}
$$


for all $X>1$. Thus we have $Y_{E}^{+}>Y_{3}^{+}$for $X>1$. In a similar way we can compare $Y_{E}^{+}$to $Y_{5}^{+}$

$$
\begin{aligned}
Y_{5}^{+}-Y_{E}^{+} & =\sqrt{\frac{24 X^{3}-15 X^{2}-42 X+33}{16}}-\sqrt{\frac{4 X^{3}+5 X^{2}-8 X}{4}}-\frac{X+1}{4} \\
& >\sqrt{3 X^{3} / 2-X^{2}-3 X}-\sqrt{X(X+5 / 8)^{2}}-X / 2 \\
& =\sqrt{X}\left(\sqrt{3 X^{2} / 2-X-3}-(X+\sqrt{X} / 2+5 / 8)\right) \\
& >\sqrt{5 X^{2} / 4+\left(X^{2} / 4-X-3\right)}-(X+\sqrt{X} / 2+5 / 8) \\
& >\sqrt{5} X / 2-(X+\sqrt{X} / 2+5 / 8) \\
& >X / 9-\sqrt{X} / 2-5 / 8 \\
& >0
\end{aligned}
$$

for all $X>36$. Thus we have $Y_{3}^{+}<Y_{E}^{+}<Y_{5}^{+}$for all $X>36$.

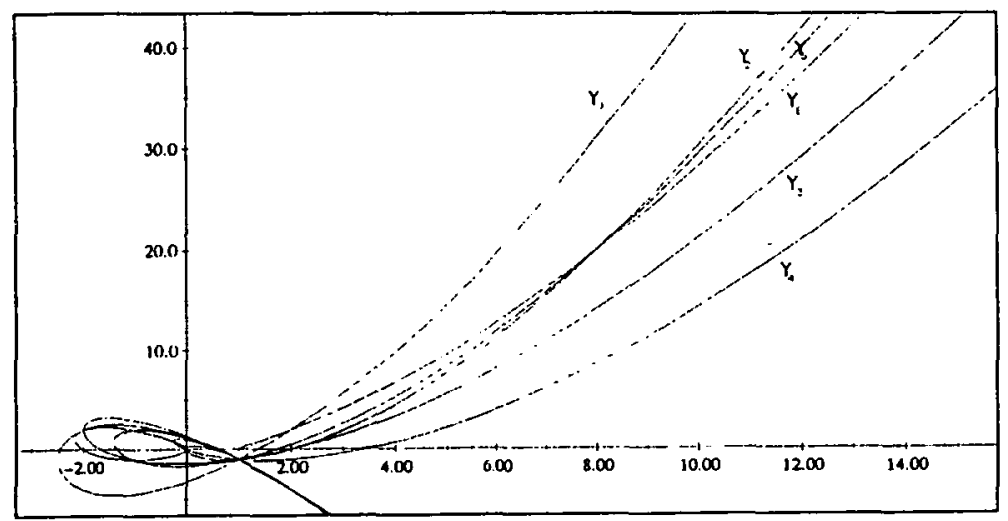

Figure 2: The graphs of $Y_{1}, Y_{2}, Y_{3}, Y_{4}, Y_{5}$ and $Y_{E}$

Using Lemma 5 and the fact that $(2,2)$ is an infinite order point on the unbounded part of $E$ we conclude that there are infinitely many rational points $(X, Y) \in E(\mathbb{Q})$ such that $X>36$. This proves the theorem.

\section{New Sporadic Triangle}

Since the completion of the proof of the main result of this paper we have continued a systematic search for more sporadic Heron triangles with two rational medians and 
have recently been rewarded for our perseverence with a new one, namely the last one shown in Table 1.

Table 1. Sporadic Heron-2-median triangles

\begin{tabular}{rrrcccr}
\hline & Sides & & & Medians & Area \\
$a$ & $b$ & $c$ & $k$ & $l$ & \\
\hline 4368 & 1241 & 3673 & 1657 & $7975 / 2$ & 2042040 \\
14791 & 14384 & 11257 & $21177 / 2$ & 11001 & 75698280 \\
2288232 & 1976471 & 2025361 & 1641725 & $3843143 / 2$ & 1877686881840 \\
\hline
\end{tabular}

The interest in these triangles is due to the fact that none of them lie on any of the 5 elliptic curves we have discovered so far and hence may lead to a new infinite family of such triangles.

\section{CONCLUSION}

It seems likely, at least to the authors, that none of the Heron-2-median triangles generated from these elliptic curves actually have 3 rational medians. This belief is supported by the observation that the half angle cotangent of the angle at the vertex of the third median is expressible in terms of two so-called 'Somos sequences' (see [2]) and is irrational in each case calculated thus far. Furthermore, we have checked the first 100 multiples of the infinite order point on $E$ (both with and without adding the order 2 point) and none of the corresponding triangles has a third rational median.

\section{REFERENCES}

[1] R.H. Buchholz, On triangles with rational altitudes, angle bisectors or medians, (Doctora] Dissertation) (Newcastle University, New South Wales, 1989).

[2] R.H. Buchholz and R.L. Rathbun, 'An infinite set of Heron triangles with two rational medians', Amer. Math. Monthly 104 (1997), 107-115.

[3] J.E. Cremona, Algorithms for modular elliptic curves (Cambridge University Press, Cambridge, 1992).

Department of Defence

Defence Science and Technology Organisation

Locked Bag 5076

Kingston ACT 2604

Australia

e-mail: ralph@defcen.gov.au
403 Marcos St Apt C

San Marcos CA 92069-1509

United States of America

e-mail: randall_rathbun@rc.trw.com 